\title{
Myometrial vascular damage after surgical sterilisation by tubal diathermy
}

\author{
G. TREGSON-ROBERTS, S. A. HANLEY, AND J. T. ROBERTS \\ From the Department of Laboratory Medicine, Henderson General Hospital, and Faculties of Medicine \\ and Pathology, McMaster University, Hamilton, Ontario, Canada
}

SUMMARY Vascular damage is described in the myometrium adjacent to areas of previous tubal diathermy for sterilisation. Elastosis, different in degree from that seen in the multiparous uterus, is most common, but more striking is medial arteriolar muscle loss with microaneurysm formation and occasional rupture.

Laparoscopy with tubal diathermy is now an accepted method of sterilisation for women (Whitehouse, 1971). An increased incidence of menorrhagia is reported after this procedure but its mechanism is not clear (Neil et al., 1975). We have examined a series of $\mathbf{4 3}$ uteri from patients in whom hysterectomy had been performed subsequent to diathermy and have rarely noticed any gross pathology. On microscopy, however, we have seen vascular changes near the diathermy burn site, which may prove to be significant.

\section{Material and methods}

The material for study consists of uteri submitted to an anatomical pathology service with a variety of stated indications for hysterectomy. Routinely, sections were taken from the cervix, endometrium, myometrium, and, when present, appendages. Conventional histological techniques were used with formalin fixation and paraffin embedding.

\section{SPECIAL BLOCKS}

Special blocks were taken from the superolateral uterine angle so as to include the superficial portion of the intramural tube. They were cut from the serosa into the myometrium parallel to the plane of the uterine cavity. The point of penetration of the fallopian tube or the diathermy scar was used as a guide. All blocks were stained with haematoxylin and eosin and Verhoff van Gieson for elastic tissue. Step and serial sections were cut as required.

After the microscopical examination the patients'

Received for publication 7 December 1977 medical records were inspected, and information from these is given in the Table.

Table Information obtained from medical records

\begin{tabular}{lllc}
\hline & $\begin{array}{l}\text { Previous } \\
\text { diathermy }\end{array}$ & $\begin{array}{l}\text { Previous } \\
\text { tubal ligation }\end{array}$ & $\begin{array}{c}\text { No previous } \\
\text { sterilisation }\end{array}$ \\
\hline No. of patients & 43 & 10 & 30 \\
Mean age & 35.7 & 32.9 & 43 \\
$\begin{array}{l}\text { Mean parity } \\
\begin{array}{l}\text { Mean } \\
\text { preoperative }\end{array}\end{array}$ & 3.0 & $2 \cdot 6$ & 3.0 \\
$\begin{array}{l}\text { haemoglobin } \\
\text { (g/dl) }\end{array}$ & 12.5 & $12 \cdot 1$ & 12.8 \\
$\begin{array}{l}\text { No. with } \\
\text { menorrhagia }\end{array}$ & 26 & & \\
\hline
\end{tabular}

\section{Results}

In the 43 test cases the site of the diathermy burn was almost always visible as a tiny serosal scar with a little radiation of the surrounding vessels. The most interesting findings on microscopy were seen in two cases where there had been a three-month interval between diathermy and hysterectomy. Both showed areas of medial muscle loss in a myometrial arteriole with aneurysmal bulging of the residual elastica (Fig. 1). In one case (Fig. 2) an adjacent portion of the same vessel showed rupture with microdissection of blood into the coat of the vessel.

Both these cases, and a third case with a similar, short diathermy-hysterectomy interval, showed areas of eosinophilic myometrial necrosis. These were surrounded by histiocytes to produce a palisading effect. We have previously observed somewhat larger areas of granulomatous necrosis, which we ascribe to the effects of diathermy (Roberts et al., 1977). 


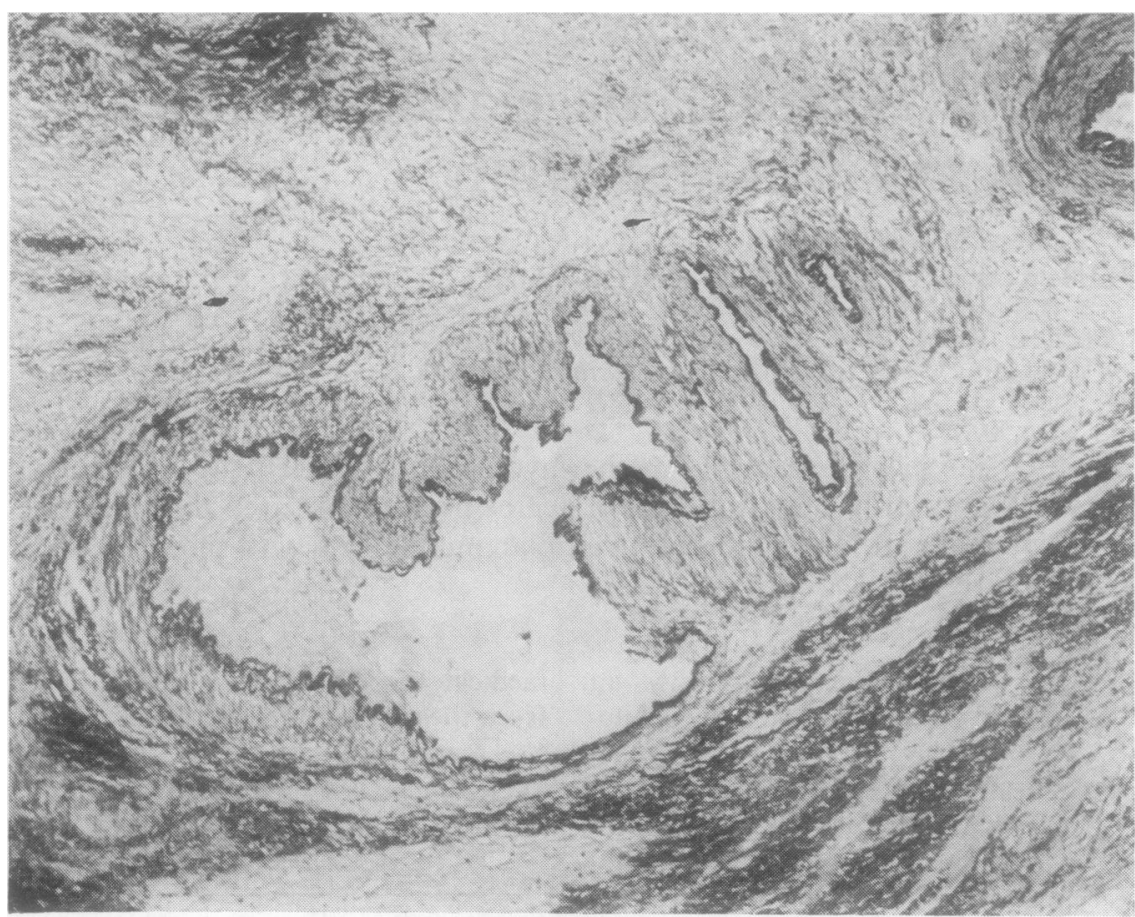

Fig. 1 Focal medial muscle loss with bulging of arteriolar wall three months after diathermy. Verhoff van Gieson $\times 50$.

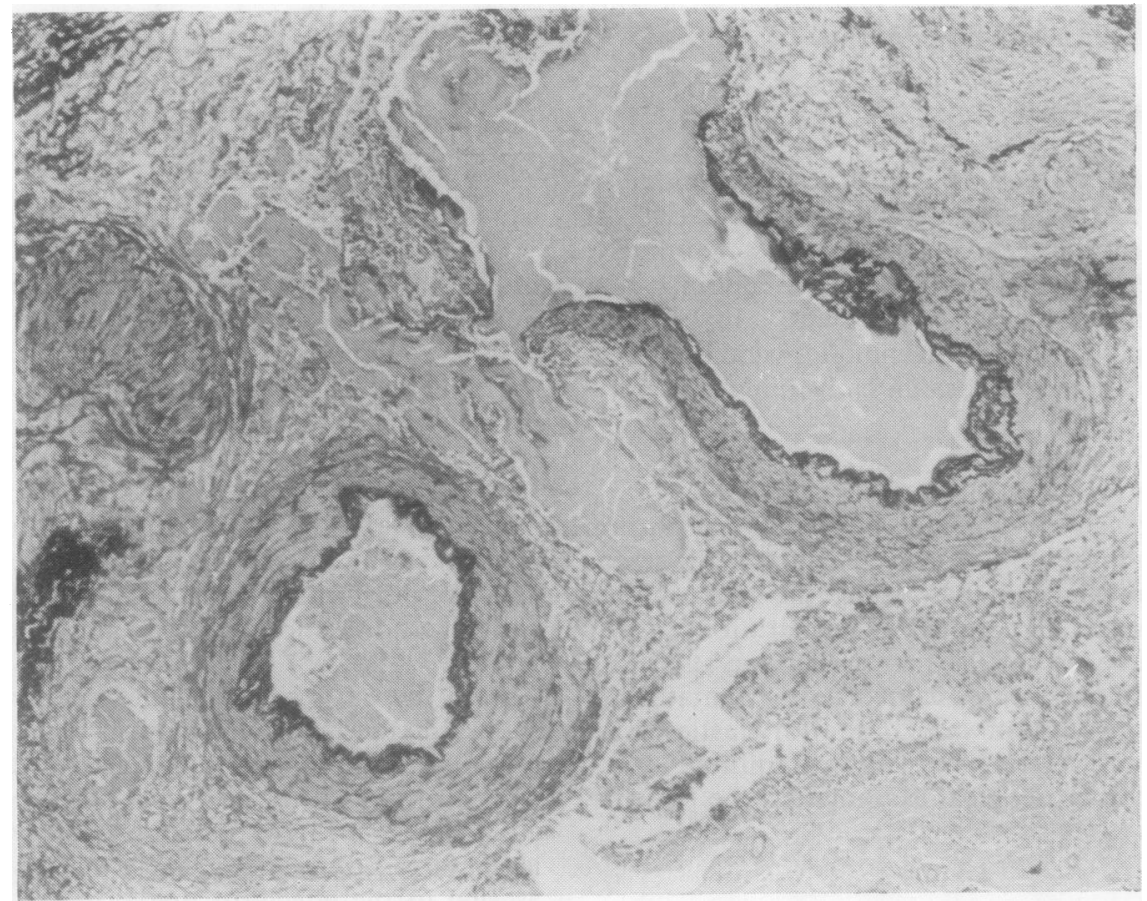

Fig. 2 Arteriolar wall rupture with perivascular haemorrhage. Same case as in Fig. 1. Verhoff van Gieson $\times 50$. 
In the remaining six cases where the diathermyhysterectomy interval was less than two years, and in about one-third of the cases with a longer interval, further characteristic changes could be seen. Haematoxylin and eosin stained sections showed masses of pink material adjacent to small cysts. These cysts were lined by columnar epithelium and superficially resembled endometriosis. Tracing by serial sections revealed them to be the lining of tubal residua. In the small zone between completely ablated tube and normal, the tube muscle seemed to disappear before the epithelium.

Elastic stains revealed the rather inconspicuous pink material in haematoxylin and eosin to be a

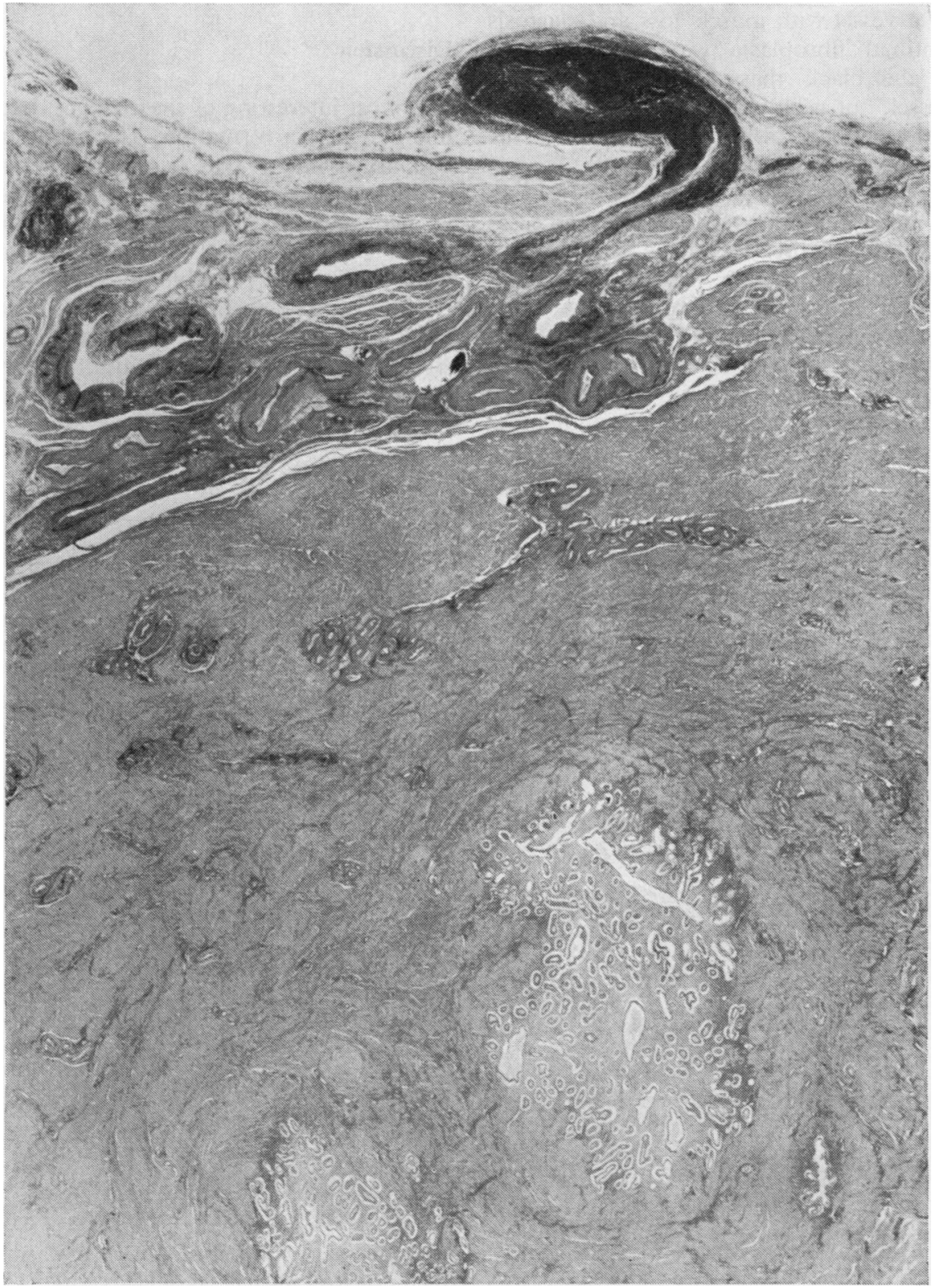

Fig. 3 Scanning view shows sharply localised (black) elastosis in the subserosa whereas deeper vessels are unaffected. Endometrium is seen below. Verhoff van Gieson $\times 4$. 
prominent hyperplastic elastosis. This was best observed by scanning/lens (Fig. 3), when the large black mass contrasted conspicuously with unaffected vessels deeper in the myometrium. Often the elastosis obviously involved the wall of a vessel, and both arteries and veins may have been affected. In larger vessels the change was often quite sharply demarcated and occasionally started at a bifurcation (Fig. 4). Sometimes the damage appeared to involve only part of a vessel with muscle loss and elastosis (Fig. 5). Intimal fibroplasia was also seen. In other cases the black mass did not obviously involve a vessel, but usually high power showed a tiny, central, endothelial-lined channel in the middle of the fibrillar material.

Examination of the medical records of these cases was not very helpful. The two most interesting cases had menorrhagia but this may have been present before diathermy. When the nine cases with a diathermy-hysterectomy interval of less that two years were taken together they did not produce any obvious pattern of symptoms, and we feel that retrospective analysis has a limit at this point. Specifically, this last group showed no endometrial abnormalities but one had adenomyosis and two hado pelvic adhesions noted by the surgeon.

The control cases and the cases with tubal ligations? did not show these vascular changes except foro elastosis related to parity, which was equally pres-0ㅡㅁ ent in test and control cases. The present form of elastosis can be distinguished by its site, amount $\stackrel{\mathbb{\Omega}}{\Omega}$ focal character, and the associated changes described above.

\section{Discussion}

The most interesting of the changes described is the microaneurysm formation. The localisation enablesi one to contend that it is related to previous diathermy. Selective passage of current along vesselso is mentioned in the older literature (Jaffe, 1928), and a study of vessels of accidental and judiciab electrocution reveals blood vessel damage with rupture and detachment of the internal elastice lamina (Hassin, 1937; Haase and Luhan, 1959) Buckley (1960) produced intimal damage undere experimental conditions with high-frequency current.

Coagulation diathermy is said to use an alternat

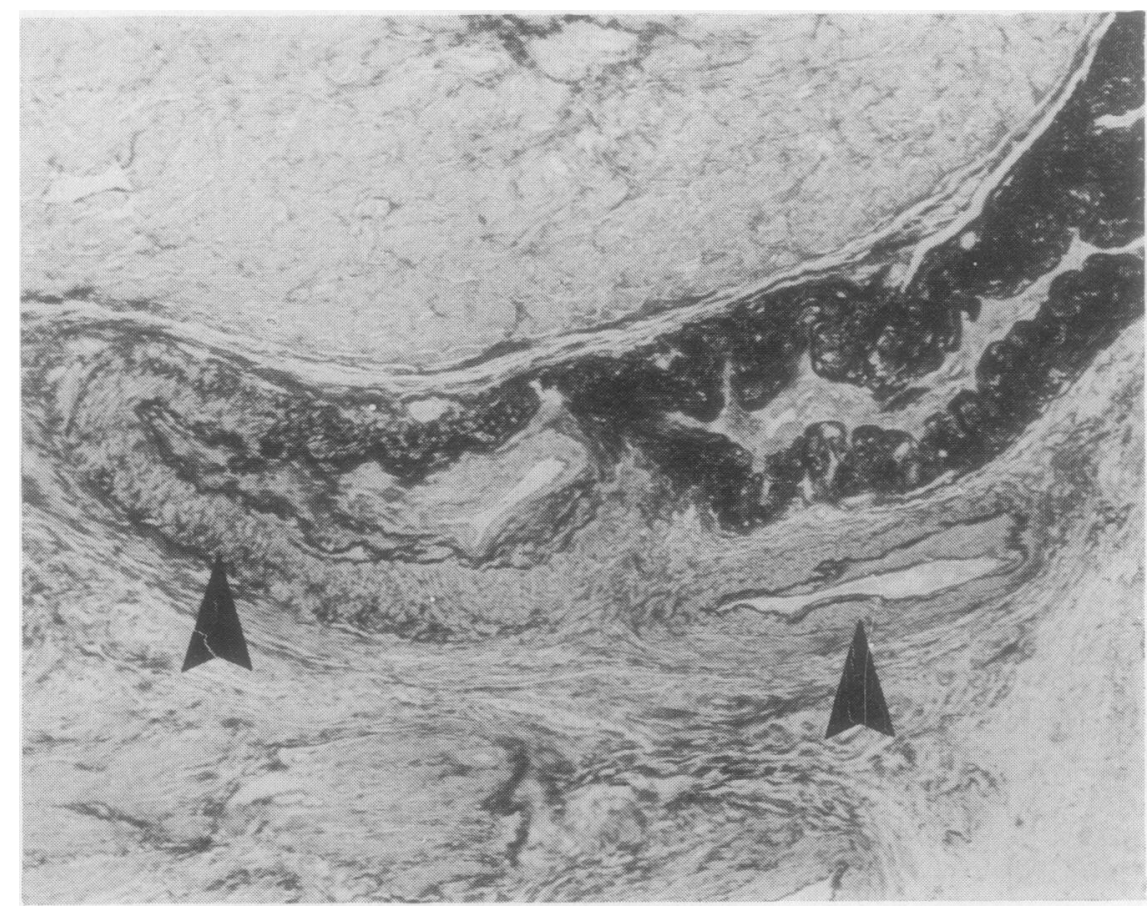

Fig. 4 Elastosis starting at a vascular bifurcation. Arrows point to separate parts of a vessel that proved to be continuous on serial section. Material staining black (for elastic tissue) is continuous with internal elastic lamina of main vessel and appears to represent a damaged side branch. Verhoff van Gieson $\times 50$. 


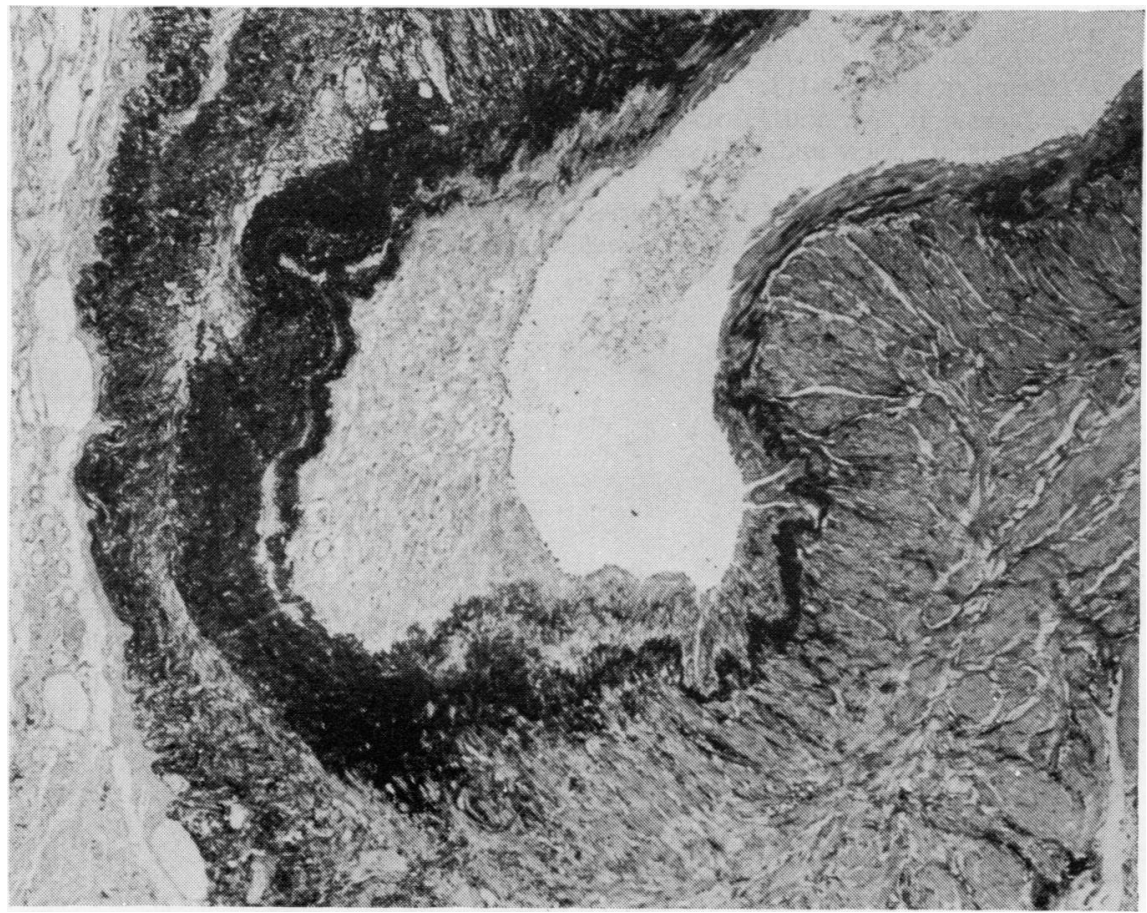

Fig. 5 A myometrial vein shows segmental damage. There is medial muscle loss (left) with replacement elastosis. There is intimal fibroplasia. On the right the wall is relatively normal. This type of change is not specific for diathermy but is seen more often and is more marked. Verhoff van Gieson $\times 50$.

ing current with a radio frequency of about $400 \mathrm{kHz}$ and a voltage of up to 5000 (Bond, 1975). A burn is produced by heat generation related to resistance to the passage of the current. The heating effect varies at the metal/tissue contact with the area of the electrode (Robinson et al., 1965; Schwimmer, 1974).

Elastosis, the production of fibrillar material with the staining characteristics of elastic tissue, is seen in diverse situations, such as sun-damaged skin, with certain tumours (carcinoma of the breast, carcinoid) and in the parous uterus. This would suggest that a variety of stimuli may cause it.

Diathermy obviously produces a local burn with death and shrinkage of tissue. We suggest that peripheral to this area of complete tissue destruction there may be a zone where current travels along the vessels. This may produce acute vascular damage with preferential muscle loss. We would view the elastosis as a nonspecific reaction of vessels that are known to show this change after other stimuli.

We make no claim to have demonstrated a relationship to bleeding. There is a limit to the value of retrospective examination of case histories, and this matter would have to be settled by a separate prospective study.

\section{References}

Bond, W. H. (1975). Electrical hazards of disposable monitoring electrodes (Letter). Lancet, 1, 852-853.

Buckley, I. K. (1960). Tissue injury by high frequency electric current. Observations with the SandisonClark ear-chamber. I. Injury to small vessels. Australian Journal of Experimental Biology, 38, 195-210.

Haase, E., and Luhan, J. A. (1959). Protracted coma from delayed thrombosis of basilar artery following electrical injury. Archives of Neurology, 1, 195-202.

Hassin, G. B. (1973). Changes in the brain in accidental electrocution. Journal of Nervous and Mental Disease, 86, 668-673.

Jaffe, R. H. (1928). Electropathology. A review of the pathologic changes produced by electric currents. Archives of Pathology, 5, 837-870.

Neil, J. R., Hammond, G. T., Noble, A. D., Rushton, L., and Letchworth, A. T. (1975). Late complications of sterilization by laparoscopy and tubal ligation. Lancet, 1, 699-700.

Roberts, J. T., Roberts, G. T., and Maudsley, R. F. 
(1977). Indolent granulomatous necrosis in patients with previous tubal diathermy. American Journal of Obstetrics and Gynecology, 129, 112-113.

Robinson, D. W., Masters, F. W., and Forrest, W. J. (1965). Electrical burns; a review and analysis of 33 cases. Surgery, 57, 385-390.

Schwimmer, W. B. (1974). Electrosurgical burn injuries during laparoscopy sterilization. Obstetrics and
Gynecology, 44, 526-530.

Whitehouse, D. B. (1971). Sterilization of young wives (Letter). British Medical Journal, 2, 707.

Requests for reprints to: Dr J. T. Roberts, Department. of Laboratory Medicine, Henderson General Hospitaf Concession Street, Hamilton, Ontario, L8V 1C3, Canad昂 